\title{
General Psychiatry Correlation of hair risperidone concentration and serum level among patients with schizophrenia
}

\author{
Xiujia Sun, ${ }^{1}$ Lihua Wang, ${ }^{2}$ Fuzhong Yang, ${ }^{2}$ Juanjuan Ren, ${ }^{1}$ Ping Jiang, ${ }^{1}$ \\ Hongmei Liu, ${ }^{1}$ Huafang Li, ${ }^{1}$ Chunbo Li, ${ }^{1}$ Chen Zhang ${ }^{1}$
}

To cite: Sun X, Wang L, Yang $F$, et al. Correlation of hair risperidone concentration and serum level among patients with schizophrenia. General Psychiatry 2019;32:e100042. doi:10.1136/ gpsych-2018-100042

XS and LW contributed equally.

Received 30 November 2018 Revised 06 January 2019 Accepted 09 January 2019

Check for updates

(c) Author(s) (or their employer(s)) 2019. Re-use permitted under CC BY-NC. No commercial re-use. See rights and permissions. Published by BMJ.

${ }^{1}$ Shanghai Key Laboratory of Psychotic Disorders, Shanghai Mental Health Center, Shanghai Jiaotong University School of Medicine, Shanghai, 200030, China

${ }^{2}$ Shanghai Mental Health Center, Shanghai Jiaotong University School of Medicine, Shanghai, 200030, China

Correspondence to Dr Chen Zhang;

zhangchen645@163.com; Dr Chunbo Li; licb@smhc.org.cn

\section{ABSTRACT}

Background Risperidone (RSP) has a rapid onset in vivo, low dosage and high plasma protein binding rate, therefore therapeutic drug monitoring (TDM) is needed to ensure safety in clinical treatment. However, compared with blood, hair is non-invasive, safe, non-infectious and easy to transport and store.

Aims This study aims to investigate the correlations among the drug concentrations of RSP in hair and serum, which provides an experimental basis to explore hair as a novel biomaterial to meet the needs of clinical detection. Methods 34 patients with schizophrenia treated with RSP for more than 3 months were enrolled in this study. About $1 \mathrm{~cm}$ section of hair near the scalp was taken from the subjects, pretreated and detected by liquid chromatography-mass spectrometry. A correlation analysis was conducted among the drug concentrations in hair, the serum concentrations and the daily dosage. The data were analysed using SPSS 20.0 software.

Results There was significant correlation between the hair concentration of RSP (two-tailed test, $r=0.440$, $p=0.009$ ) with the serum concentration of RSP, and the hair concentration of 9-hydroxyrisperidone (9-HR) with the serum concentration of $9-\mathrm{HR}$ had no significant correlation (two-tailed test, $r=-0.217, p=0.217$ ); the total concentration of the RSP and 9-HR had no significant correlation between hair and serum $(r=0.227, p=0.196)$. The dosage had no statistically significant correlation with the concentration of RSP in hair $(r=0.207, p=0.241), 9-H R$ in hair $(r=-0.194, p=0.271)$ and the total concentration of RSP and 9-HR in hair ( $r=0.188, p=0.288)$. There was no statistical correlation between the dosage and the concentration of RSP in serum ( $r=-0.059, p=0.741)$, but significant correlation between the dosage and 9-HR in serum $(r=0.581 p<0.001)$ was found, and the correlation between the dosage and the total concentration of the two drugs RSP and 9-HR in serum was also significant $(r=0.437, p=0.01)$.

Conclusion The correlation analysis showed that the concentration of RSP in hair was statistically significant with the serum RSP concentration. In this study, we provided some experimental basis for hair as a new biomaterial to monitor the therapeutic drug concentration.

\section{INTRODUCTION}

Risperidone (RSP), a second-generation antipsychotic that antagonises the D2/5-HT2A receptor, has a good therapeutic effect on both positive and negative symptoms of schizophrenia, showing fewer adverse reactions (eg, inhibition of motor function). Thus, RSP has been widely used in clinical practice. ${ }^{1}$ RSP is metabolised in vivo by 2D6, cytochrome P450 enzyme or transporter, ${ }^{2}$ or undergoes a binding reaction via two-phase metabolism. The main metabolite of RSP, 9-hydroxyrisperidone (9 hour), also has strong pharmacological activity. The therapeutic range of 20-60 ng/mL (approximately $50-140 \mathrm{nmol} / \mathrm{L}$ ) for the active moiety (RSP +9 -HR) is often cited. ${ }^{3}$ Although the pharmacological effect is exerted by the unbound fraction of the drug, it is customary to measure the total (bound+unbound) concentration according to the previous studies. The total blood concentration of RSP and its metabolites are related to curative effect of the drug. ${ }^{4}$ Due to the narrow therapeutic window of these drugs, most antipsychotics usually cause unpleasant side effects and severe poisoning after overdose. Suicide and suicide attempts are also frequent in populations using antipsychotics $^{5}$ and several intoxications have been published. ${ }^{6} 7$ Therefore, therapeutic drug monitoring (TDM) of risperidone was used to guide treatment usually. Considering that patients tend to take several drugs simultaneously during the treatment, about 2-3 or even more blood samples $(5 \mathrm{~mL})$ are collected for detection in clinical practice. Consequently, finding new non-invasive alternative biomaterials to replace blood has widespread application value.

As an appendage of skin, hair is composed of keratinised epidermal cells. Drugs enter hair through blood circulation. Hair is easier to store than blood and is not easily affected by the ambient temperature, serving as a good biological sample. Moreover, hair is safe, stable and non-infectious without restrictions of time and place, which could avoid 
35 patients with schizophrenia met the following criteria:

(1) Patients with schizophrenia who were treated with RSP for more than 3 months;

(2) Participants who were willing to participate in this study signed the informed consent;

(3) Participants who have black hair;

(4) No limitation to gender.

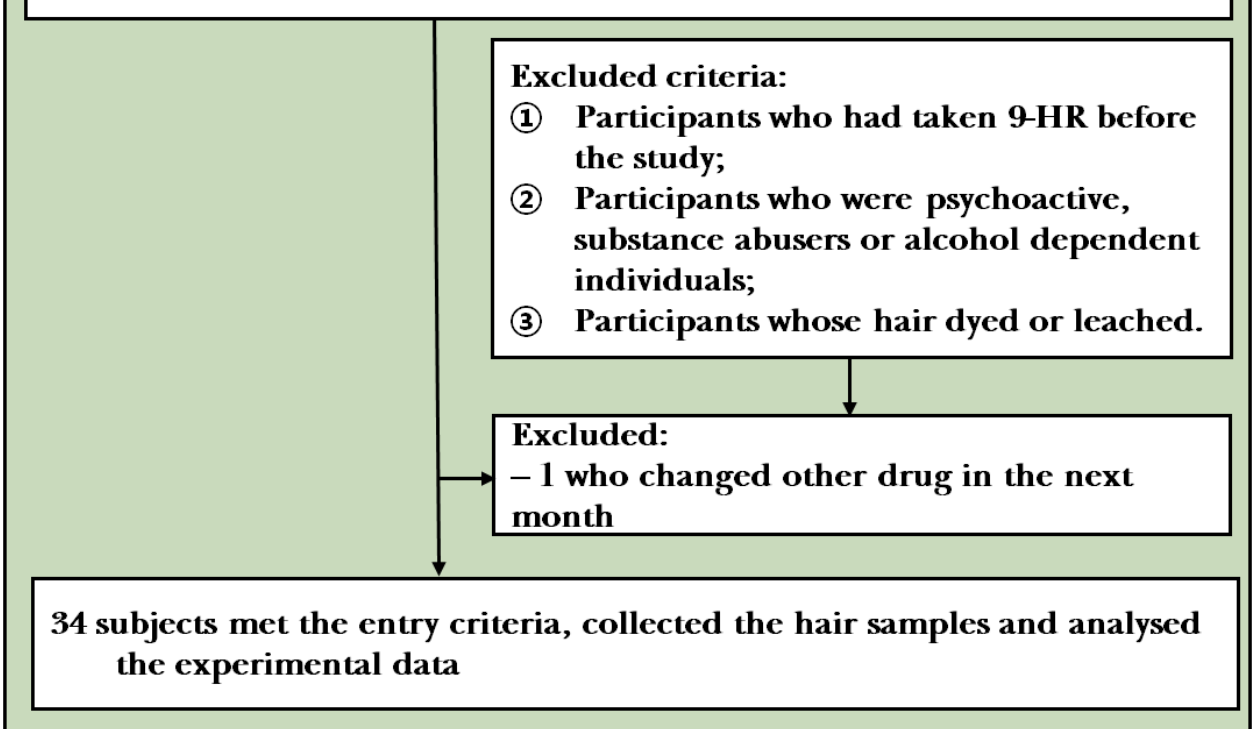

Figure 1 Flowchart of the study

physical discomfort, dizziness and infection caused by blood drawing. ${ }^{8}$ Hair analysis can also offer the possibility of revealing an individual's history of drug exposure for periods varying from weeks to months, depending on the length of the hair shaft. ${ }^{9}$ Segmental hair has been used to detect the concentrations of RSP and 9-HR in a study previously. ${ }^{10}$ There were only three cases in the study, and the correlation between the concentration of RSP in hair and in blood was also not further studied. In our preliminary work, a liquid chromatography-mass spectrometry (LC-MS/MS) method was established to detect the concentration of RSP and 9-HR in hair. ${ }^{11}$ Moreover, it was found that the concentration of RSP in hair at the $2 \mathrm{~cm}$ segment near the scalp was well correlated with the dosage. At present, however, the correlation between the concentrations of RSP and 9-HR in hair and their blood concentrations remains unclear. We conducted a preliminary study to investigate the correlation between the concentration of RSP in the hair of hospitalised patients and the historical blood concentration, providing an experimental basis for the new testing method and biological samples. The flowchart can be referred in figure 1 .

\section{MATERIALS AND METHODS}

\section{Participants}

The participants met the following inclusion criteria: (1) patients with schizophrenia who were treated with RSP for more than 3 months; (2) participants who were willing to participate in this study provided written informed consent before enrolment; (3) participants who have black hair; (4) not limited to gender. The participants were excluded according to the following exclusion criteria: (1) participants who had taken 9-HR before the study; (2) participants who were psychoactive substance abusers or alcohol dependent individuals; (3) participants whose hair was permed, dyed or bleached. The exit criteria were as follows: (1) participants who did not agree to continue or participate in the study (withdrawal of informed consent); (2) participants who were not considered to meet the requirements of the study (the administration time was uncertain or with no history of blood concentration data); (3) participants who were lost to follow-up. The hair was collected at 07:00 a.m., and the steady-state minimum concentration about 12 hours after the last administration was regarded as the history of blood concentration.

\section{The sampling and pretreatment of hair}

By reviewing their medical history, we recorded the history of blood concentration data in the corresponding month, dosage and combined medication of patients with schizophrenia who took fixed-dose RSP for more than 3 month. Total 35 patients who met the enrolment criteria were included. One patient was excluded due to the use of other drugs during the third month. Thus, 34 patients were actually included into the study. 
The enrolled patients were screened according to the inclusion criteria and an area of $1 \mathrm{~cm}^{2}$ of hair (about the thickness of a small finger thickness) near the scalp area of occipital region was taken. The root position of hair was marked. The samples were enclosed in envelopes, numbered and stored in a dry environment at room temperature.

Determination of the concentrations of RSP and 9-HR in hair Pretreatments of hair included washing, grinding, dissolving, extracting and purifying were performed. The hair samples of each patient were numbered, washed with distilled water, acetone and distilled water again and dried in the air. The hair near the root segment was cut into $1 \mathrm{~cm}$ pieces and grinded. About $20 \mathrm{mg}$ hair was accurately weighed and transferred into a $10 \mathrm{~mL}$ centrifuge tube with a lid. The internal standard working solutions, including deuterated risperidone (RSP- $\mathrm{d}^{4}, 1.0 \mu \mathrm{g} / \mathrm{mL}$ ) and deuterated 9-hydroxyrisperidone (9-HR- $\mathrm{d}^{4}, 0.01 \mu \mathrm{g}$ / $\mathrm{mL})$ solutions, were prepared. Then, $100 \mu \mathrm{L}$ RSP-d $\mathrm{d}^{4}, 50$ $\mu \mathrm{L}$ 9-HR-d ${ }^{4}$ and $1 \mathrm{~mL}$ sodium hydroxide solution $(1 \mathrm{~N})$ were added to the centrifuge tube. After a supersonic treatment for 2 hours, the mixed solution was neutralised by an equal volume of hydrochloric acid $(1 \mathrm{~N}), 200 \mu \mathrm{L}$ of $1 \mathrm{~N}$ sodium hydroxide was added to adjust the $\mathrm{pH}$ value. To the mixed solution, $5.0 \mathrm{~mL}$ methyl tert-butyl ether was added. Then, the solution was mixed by vortex-mixing for $1 \mathrm{~min}$, and the supernatant was transferred to a new tube and evaporated to dryness under a stream of nitrogen at $40^{\circ} \mathrm{C}$ water bath. The residue was redissolved in $100 \mu \mathrm{L}$ mobile phase, and $5 \mu \mathrm{L}$ supernatant was injected into LC-MS/MS.

Chromatographic conditions: Chromatographic separation was achieved on an Agilent Zorbax $\mathrm{SB} \mathrm{C}_{18}$ column $(2.1 \times 50 \mathrm{~mm}, 1.8 \mu \mathrm{m})$ at $30^{\circ} \mathrm{C}$ using isocratic elution with mobile phase. The mobile phase consisted of water $(\mathrm{pH}$ 4.0 was adjusted by formic acid) containing ammonium acetate $(0.01 \mathrm{~mol} / \mathrm{L})$ and acetonitrile $(70: 30 \mathrm{v} / \mathrm{v})$. The flow rate was set at $0.3 \mathrm{~mL} / \mathrm{min}$. A $5 \mu \mathrm{L}$ sample solution was injected into the LC-MS/MS system for analysis. The mass spectrometer was performed on electrospray ionisation source $(\mathrm{ESI}+)$ by multiple reaction monitoring (MRM) mode. Nitrogen was used as nebulising and secondary gas at a pressure of 35.0 psi. The drying gas was set at a flow rate of $10.0 \mathrm{~L} / \mathrm{min}$ and the temperature was set at $350^{\circ} \mathrm{C}$. The following parameters were employed: capillary voltage (+) $4.0 \mathrm{kV}$, Delta EMV (+) $300 \mathrm{~V}$. The MRM acquisition parameters were as follows: $\mathrm{m} / \mathrm{z} 411.2 \rightarrow 191.0$ for RSP, $\mathrm{m} / \mathrm{z} 427.2 \rightarrow 207.1$ for $9-\mathrm{HR}, \mathrm{m} / \mathrm{z} 415.2 \rightarrow 195.2$ for RSP-d ${ }^{4}$ and $\mathrm{m} / \mathrm{z} 431.2 \rightarrow 211.1$ for $9-\mathrm{HR}-\mathrm{d}^{4}$.

The detail of method validation has been described previously by our group, ${ }^{11}$ and for the LC-MS/MS TIC and MRM ion chromatograms in No. 3 sample of the standard and internal standard substances, see figure 2.

\section{Statistical analyses}

SPSS V.20 software was used for the analysis. For the correlations among the dosage, the concentration of
RSP, 9-HR, the sum of the RSP and 9-HR in hair and the concentration of RSP, 9-HR, the sum of the RSP and 9-HR in serum of the corresponding month, respectively, Spearman's correlation was used.

\section{RESULTS}

\section{The determined results of the samples}

The demographic data, daily dosage of the subjects, the concentrations of RSP and 9-HR in hair, and the serum concentrations of the corresponding month are shown in table 1.

A total of 34 subjects were enrolled in our study. The correlations between dosage and the concentration of RSP, 9-HR or the sum of two drugs in the hair and the respective serum concentration were analysed.

\section{The correlations between the dosage and concentration of} RSP, 9-HR, the sum of the two drugs in serum and hair

As shown in table 2, this work showed statistically significant correlations between the concentration of RSP in hair and the serum RSP concentration (two-tailed test, $\mathrm{r}=0.440, p=0.009$ ), see figure 3 ; the concentration of 9-HR showed no statistically significant correlations between hair and serum samples (two-tailed test, $r=-0.217$, $p=0.217)$; and the total concentration of the two drugs RSP and 9-HR had no significant correlations between hair and serum samples (two-tailed test, $\mathrm{r}=0.227, p=0.196$ ).

The dosage showed no statistically significant correlations between the concentration of RSP in hair (two-tailed test, $\mathrm{r}=0.207, p=0.241$ ) and 9-HR in hair (two-tailed test, $\mathrm{r}=-0.194, p=0.271$ ), and the total concentration of the two drugs RSP and 9 -HR in hair (two-tailed test, $\mathrm{r}=0.188$, $p=0.288$ ).

There were no statistically significant correlations between the dosage and the concentration of RSP $(\mathrm{r}=-0.059, p=0.741)$, but it showed significant correlations between the dosage and the concentration of 9-HR in hair (two-tailed test, $\mathrm{r}=0.581, p<0.001$ ) and the dose showed significant difference with the total concentration of the two drugs (RSP and 9-HR) in serum $(r=0.437, p=0.010)$.

\section{DISCUSSION}

\section{Main findings}

Hair samples have been widely used in medicolegal analysis. Antipsychotics are being used from several milligrams to hundreds of milligrams daily. After a specific period of accumulation time, the content of the drug in hair is higher and the quantity of the original drug and its metabolites can be tested simultaneously. Shen ${ }^{12}$ and Doherty ${ }^{13}$ introduced the method of simultaneous detection of antipsychotics and their metabolites by gas chromatography-mass spectrometry (GC/MS) and LC/MS/ MS, respectively. Compared with the traditional GC-MS, LC/MS/MS method can be screening analysis of antipsychotic drugs in hair, and it can be adopted to the MRM mode which effectively reduces the influence of hair 

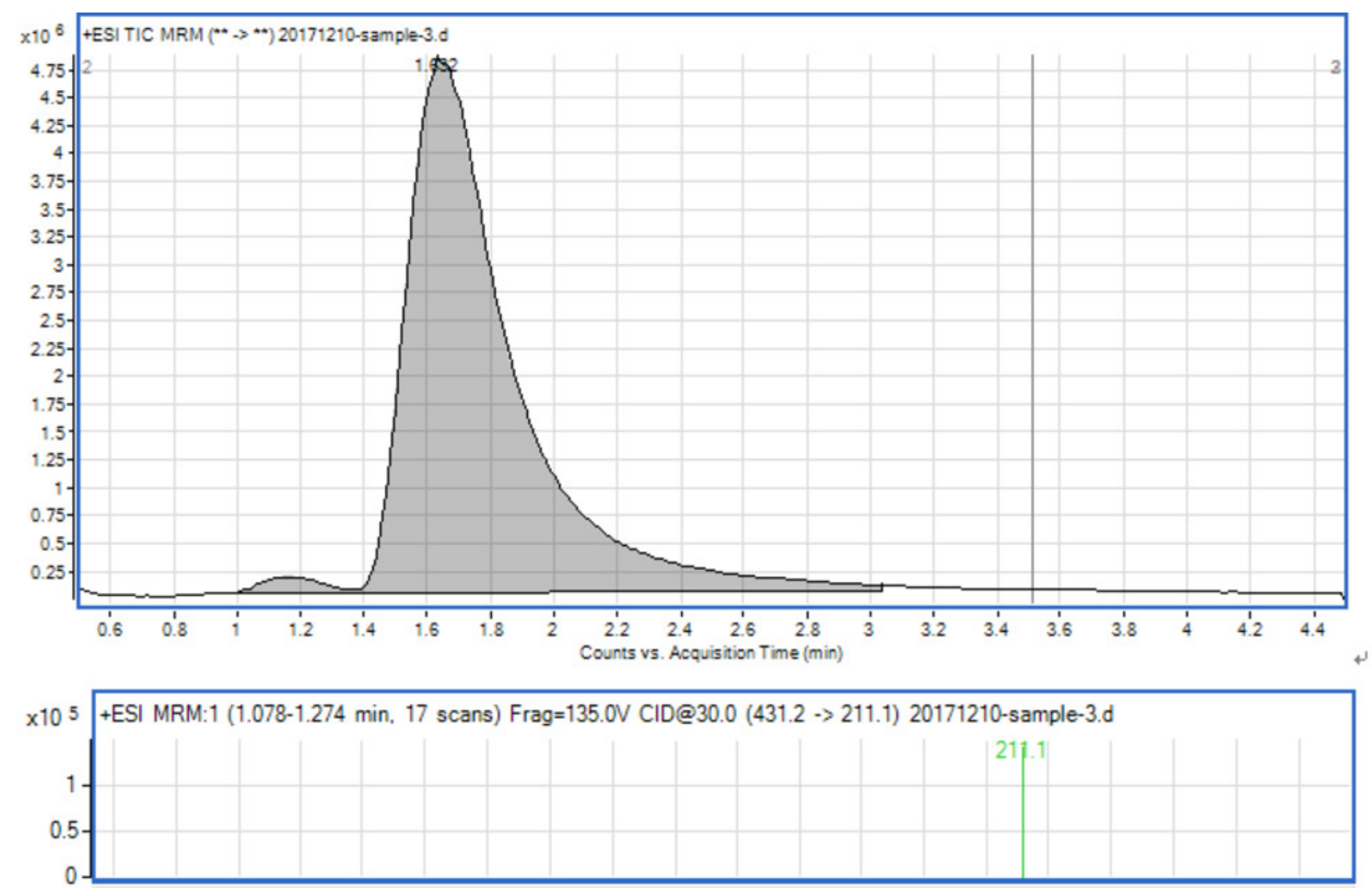

x10 4 +ESI MRM:2 (1.081-1.277 min, 17 scans) Frag=130.0V CID@20.0 (427.2 $>$ 207.1) 20171210-sample-3.d

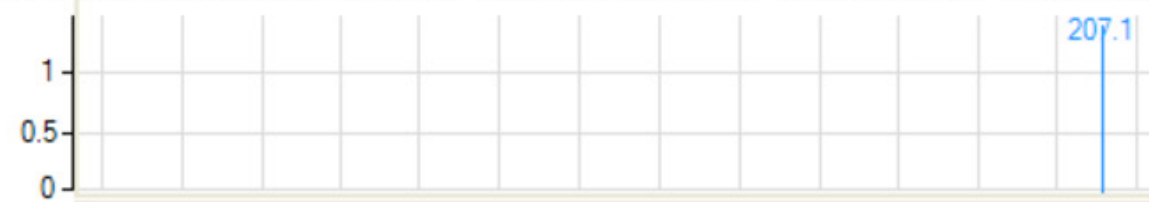

x10 4 +ESI MRM:3 (1.512-1.831 min, 27 scans) Frag=135.0V CID@25.0 (415.2 -> 195.2) 20171210-sample-3.d

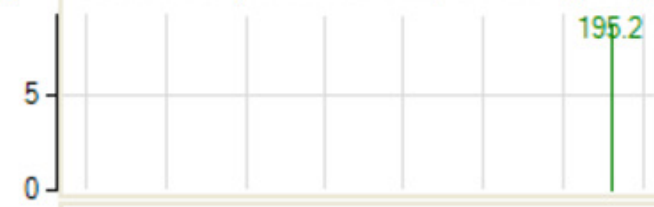

x106 +ESI MRM:4 (1.516-1.834 min, 27 scans) Frag=130.0V CID@28.0 (411.2 $\rightarrow$ 191.0) 20171210-sample-3.d

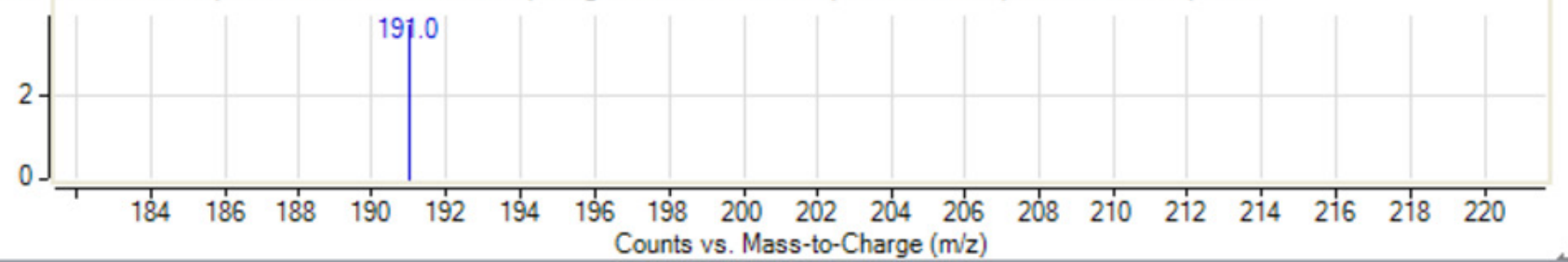

Figure 2 The LC-MS/MS TIC and MRM ion chromatograms of the RSP and 9-HR and d4 internal standard substances

matrix and greatly improves the sensitivity. It can detect up to pictogram/milligram level targets in the hair, especially highly polar and instable drugs and their metabolites. ${ }^{14} 15$ The ESI-MS ${ }^{2}$ fragment pathway of RSP and its metabolites 9-HR is shown in figure 4. Therefore, LCMS/ MS method was used to detect the RSP and 9-HR concentration of all the 34 hair samples.
Hair is a non-invasive and favourable biological marker for monitoring the long-term systemic exposures to medications. Moreover, hair grows at a rate of approximately 1 $\mathrm{cm}$ per month and drug analysis in hair may allow for the identification of metabolic activity throughout the period of medication taken medicine. In contrast, blood can only provide information regarding drug concentrations 
Table 1 Results of the concentrations of RSP and 9-HR in hair and in serum from 34 subjects

\begin{tabular}{|c|c|c|c|c|c|c|c|}
\hline & \multirow[b]{2}{*}{$\begin{array}{l}\text { Daily dosage } \\
\text { (mg/day) }\end{array}$} & \multicolumn{3}{|l|}{ Hair } & \multicolumn{3}{|l|}{ Serum } \\
\hline & & RSP (ng/mg) & 9-HR (ng/mg) & $\begin{array}{l}\text { RSP+9-HR (ng/ } \\
\mathrm{mg})\end{array}$ & RSP (ng/mL) & 9-HR (ng/mL) & $\begin{array}{l}\text { RSP+9-HR } \\
\text { (ng/mL) }\end{array}$ \\
\hline Female $(n=21)$ & $4.5(2.5-5.0)$ & $\begin{array}{l}11.8(3.50- \\
19.05)\end{array}$ & $\begin{array}{l}0.223(0.0675- \\
1.2495)\end{array}$ & $\begin{array}{l}12.967(4.106- \\
19.153)\end{array}$ & $\begin{array}{l}7.3(2.40- \\
13.25)\end{array}$ & $\begin{array}{l}23.1(14.5- \\
38.1)\end{array}$ & $\begin{array}{l}30.6(21.2- \\
42.8)\end{array}$ \\
\hline
\end{tabular}

9-HR, 9-hydroxyrisperidone; RSP, risperidone.

at the time of sampling. Therefore, it is important to analyse the correlation between the total concentration of the two drugs in hair and in blood. However, there is no domestic study on the correlations between the concentration of RSP, 9-HR or the total of the two in hair and the corresponding concentration in blood, which we have first studied to explore whether the hair could be used as a substitute biomaterial for blood.

RSP is mainly metabolised to the active metabolite 9-HR by cytochrome P450(CYP) 2D6 in the liver. ${ }^{16}{ }^{17}$ Due to the blood-brain barrier, the concentration of 9-HR in blood was higher than the concentration of its parent drug. With $70 \%$ activity of its parent drug, 9 -HR shows similar pharmacological activity to RSP. ${ }^{18}{ }^{19}$ Usually the parent drug is much higher than its metabolites of all psychotropic drugs in the hair, and the proportion of the metabolites are different. The metabolites of chlorpromazine, carbamazepine and amitriptyline accounted for about $10 \%$ of the original drug in hair, and N-clozapine only exists in the hair of those with strong metabolic genre. ${ }^{20}$ In this study, we also noted that the concentration of 9-HR in hair was much lower than the concentration of RSP, which was consistent with Schneider's results. ${ }^{10}$ It might be caused by the fact that the metabolite 9-HR in blood was difficult to get into hair. The complexity of drugs into

Table 2 The Spearman correlation tests among the dosage and concentration of RSP, 9-HR, the sum of two drugs in hair and the respective serum concentration $(n=34)$

\begin{tabular}{|c|c|c|c|c|}
\hline & \multicolumn{4}{|c|}{ Serum } \\
\hline & & RSP (ng/mL) & $\begin{array}{l}\text { 9-HR } \\
\text { (ng/mL) }\end{array}$ & $\begin{array}{l}\text { Sum of } \\
\text { RSP and } \\
9-\mathrm{HR} \\
\text { (ng/mL) }\end{array}$ \\
\hline \multicolumn{5}{|l|}{ Hair } \\
\hline \multirow[t]{2}{*}{$\mathrm{RSP}$ (ng/mg) } & $r$ & $0.440^{*}$ & 0.156 & 0.234 \\
\hline & $\mathrm{p}$ & 0.009 & 0.380 & 0.182 \\
\hline \multirow{2}{*}{$\begin{array}{l}\text { 9-HR (ng/ } \\
\mathrm{mg} \text { ) }\end{array}$} & $r$ & 0.190 & -0.217 & -0.0171 \\
\hline & $p$ & 0.282 & 0.217 & 0.335 \\
\hline \multirow{2}{*}{$\begin{array}{l}\text { Sum of RSP } \\
\text { and } 9-\mathrm{HR} \\
(\mathrm{ng} / \mathrm{mg})\end{array}$} & $r$ & $0.469^{*}$ & 0.135 & 0.227 \\
\hline & $\mathrm{p}$ & 0.005 & 0.446 & 0.196 \\
\hline
\end{tabular}

${ }^{*} p<0.001$ (two-tailed test).

9-HR, 9-hydroxyrisperidone; RSP, risperidone. the hair is related to the physical and chemical properties, lipophilicity and especially the melanin content in the hair. Many reports showed that antipsychotics alkaline and lipophilic drugs are more likely to be traced and melanin is considered as the main factor affecting it. ${ }^{21}$

Some studies have reported the total blood concentration of risperidone and its metabolite is associated with curative effects of the drug. ${ }^{22}$ In this study, we found that the concentration of RSP in hair was significantly correlated with the RSP serum concentration $(r=0.440$, $p<0.01)$, and the concentration of 9-HR in hair had no correlation with the serum 9-HRconcentration $(r=-0.217$, $p>0.01$ ), which may be affected by the small hair value. Consequently, the total concentration of the two drugs in hair was correlated with their concentration in blood. ${ }^{23-25}$ We found that the total concentrations of the two drugs in hair were not related to the two drugs in blood, that may be related to the small 9 -HR value, which might be relevant to the fact that the metabolite 9-HR had difficulty in entering hair or be related to RSP producing 9-HRin blood by metabolism.

In this work, the dosage was not correlated with the blood concentration of RSP ( $\mathrm{r}=-0.059, p=0.741)$, but correlated with 9-HR $(\mathrm{r}=0.581, p=0.000)$ and the two drugs $(\mathrm{r}=0.437, p=0.010)$. These findings were contradictory with Wei $2005^{26}$ and Zhao $2012^{27}$ who found that the plasma concentrations of 9-HR or the sum of RSP and 9-HRwere more related to the daily dose than RSP. The

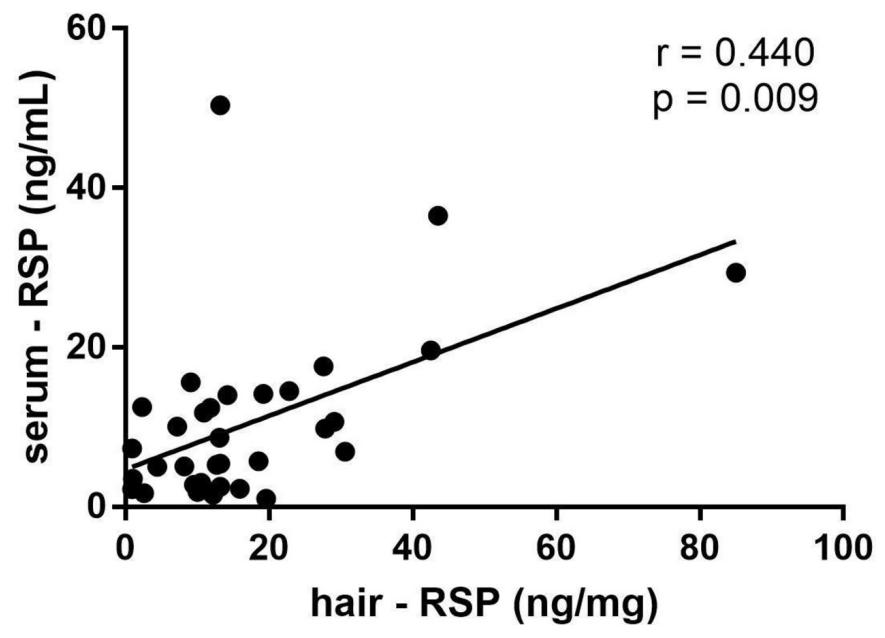

Figure 3 The correlation between the concentration of RSP in hair and in serum 

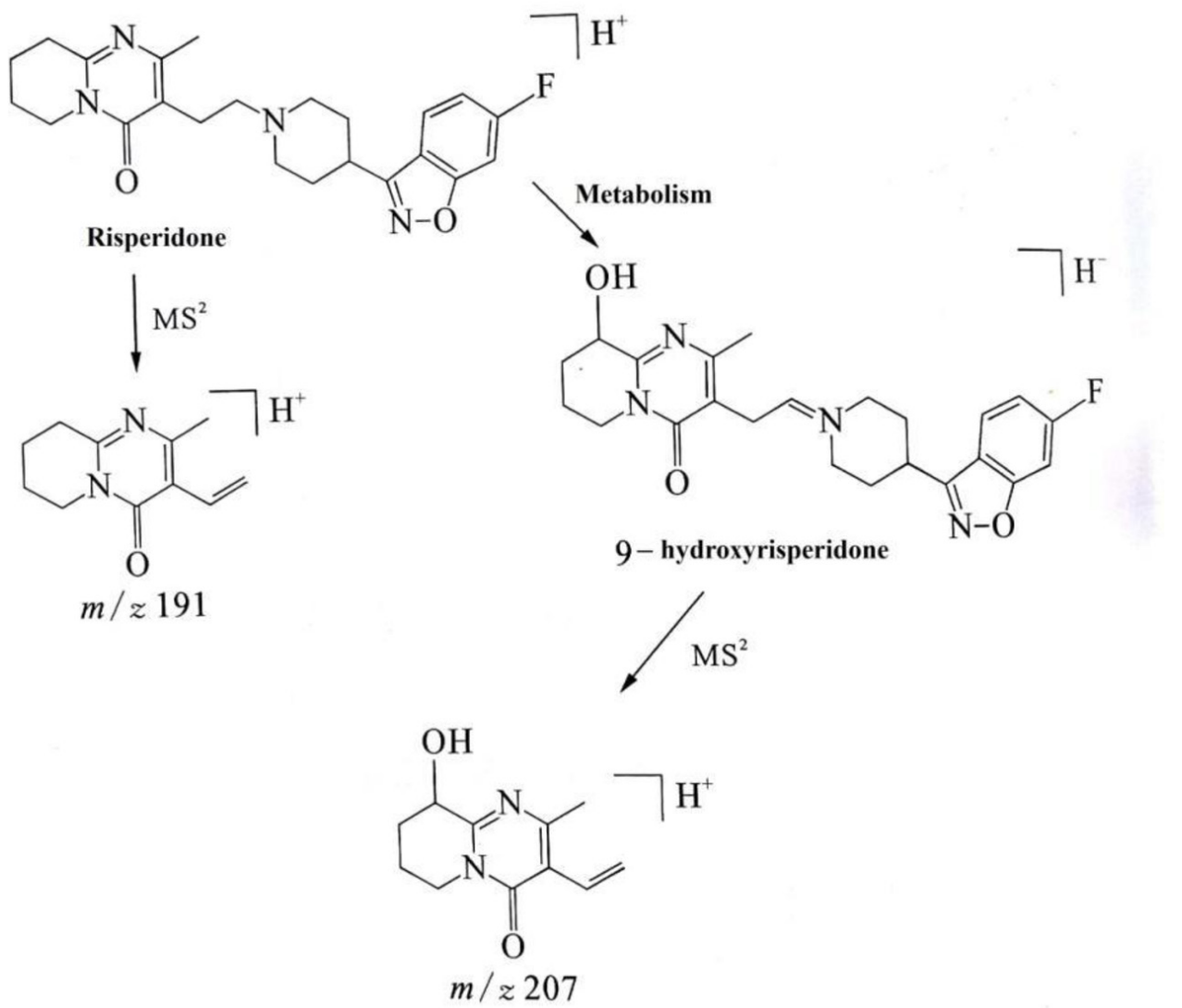

figure 4 The ESI - MS2 fragment pathway of RSP and its metabolites 9-HR

dosage had no statistically significant correlation with the concentration of RSP in hair ( $\mathrm{r}=0.207, p=0.241), 9-\mathrm{HR}$ in hair $(\mathrm{r}=-0.194, p=0.271)$ and the total concentration of RSP and 9-HR in hair $(\mathrm{r}=0.188, p=0.288)$. This may be due to the small sample size or due to the difficulty in obtaining hair samples of adequate length in male patients. It is also possible that the accumulation and metabolic mechanism in the hair is different from the mechanism in the blood. However, further research is needed.

\section{Limitations}

This study has the typical limitations of naturalistic studies, which are not designed to identify variables with small effect sizes; more controlled clinical designs are needed to identify variables with small effects. Among the subjects enrolled in this study, most of the male inpatients had a haircut once a month. The lengths of their hair samples that were collected at one time were short, which could not be divided into more segments for the measurements. Hence, the correlation between the concentrations of the other segments or the total segments and the historical blood concentration remains to be further studied.

Since the quantity of the samples in this study was not enough, there might be a statistical deviation. Accordingly, further expansion of the quantity of the samples is needed in future studies. In addition, based on ethical considerations, this study only retrospectively analysed the historical blood concentrations of the patients and determined the drug concentrations in their hair. The medications and time points have not been strictly unified. In the following studies, some prospective studies are needed, relevant data should be recorded, and hair or blood samples should be collected at different times after medication for pharmacokinetic research of drugs, but these still need further plans. With regard to taking the medicine time and blood collection time, it is best to collect blood and hair samples at different times after taking the medicine, and to fit the pharmacokinetic study in the hair, ${ }^{28}$ but this still needs further planning. Next, we may also conduct correlational studies on other psychiatric drugs between hair concentrations and blood concentrations, or even correlation studies on psychiatric drugs in combination with other drugs. In the future, we will continue to expand the types of psychiatric drugs determined by hair and continuously optimise the detection methods.

\section{Implications}

TDM refers to determining the concentrations of drugs in a biological matrix via modern analytical techniques under the guidance of pharmacokinetic principles, which is used for the guidance and evaluation of medication. ${ }^{29}$ Antipsychotics or antidepressants have a narrow therapeutic window and strong side effects and become potential pathogenic ${ }^{6}$ and lethal factors. ${ }^{30}$ Consequently, TDM is needed in clinical applications of antipsychotics and 
antidepressants as an important monitoring parameter. $^{31}$ While the collections of blood samples are invasive, they require professional operations. Blood samples should be stored and transported under special conditions, have biosafety hazards and are troublesome in waste recovery and disposal. Furthermore, plasma protein binding rate is significantly affected by individual differences. ${ }^{32} 33$ As non-invasive biological specimen, hair is easy to transport and store, is not affected by external factors and is safe, hygienic and non-infectious. ${ }^{34}$ Therefore, hair can be used as a substitute for blood, showing epoch-making significance. As a TDM sample, hair has the following advantages: the prodrugs and their metabolites in hair are stable $;^{35}$ the hair samples can be repeatedly collected; the detection window of hair can be detected for several months or even years; ${ }^{36}$ hair can be used for the information tracking of drug cessation, missing dose and drug abuse $;{ }^{37}$ the results provide the basis for diagnosis, treatment, detection of drug efficacy and patient compliance. ${ }^{38}$ As a supplement to blood and urine samples, hair shows great significance in clinical practice.

Hair analysis has been applied since the 1950s, which only was used for qualitative detection of drug exposure due to the limited testing methods at the time, ${ }^{21}$ With the development of microdetection technology in recent years, the appearance of LC-MS/MS and GC-MS has improved the detection sensitivity, making the detection of drug concentration in hair into a quantitative level. In practical applications, the drug concentration in hair can be used to verify patient compliance. ${ }^{39}$ It was found that the cortisol concentration in human hair was correlated with its blood concentration, suggesting that hair could be used as a biomaterial in vivo. However, there is no study on the correlation between the concentration of RSP in hair and its blood concentration in China. This study first found that the concentration of RSP in hair had a certain correlation with its blood concentration, which served as an experimental basis for whether hair could replace blood as auxiliary diagnosis biomaterial and provided referenced value for clinical research.

Contributors XS drafted the manuscript. LW and FZY were responsible for patient recruitment and samplings. PJ and HML provided literature and guidance for methods. JJR and XS were responsible for the determination of samples. HL provided the guidance on ethics approval. CL designed and guided this study. CZ funded the study and was responsible for the implementation of the study. The authors are responsible for the data of the paper.

Funding This work was supported by the National Natural Science Foundation of China (No. 81471358), Shanghai Health and Family Planning Commission Youth Fund Project (No. 20144Y0080), Shanghai Health and Family Planning Commission Youth Fund Project (No. 20154Y0045) and Shanghai Health and Family Planning Commission Fund Project (No. 201740089), Shanghai Natural Science Foundation(15ZR1435300); Shanghai JiaoTong University School of Medicine Natural Science and Technology Foundation(14XJ10080), the Shanghai Municipal Education Commission—Gaofeng Clinical Medicine Grant Support (No. 20152530)

Competing interests None declared.

Patient consent for publication Obtained.

Ethics approval 2013-27

Provenance and peer review Not commissioned; externally peer reviewed.
Data sharing statement No additional data are available.

Open access This is an open access article distributed in accordance with the Creative Commons Attribution Non Commercial (CC BY-NC 4.0) license, which permits others to distribute, remix, adapt, build upon this work non-commercially, and license their derivative works on different terms, provided the original work is properly cited and the use is non-commercial. See: http://creativecommons.org/ licenses/by-nc/4.0

\section{REFERENCES}

1. Laffont CM, Gomeni R, Zheng B, et al. Population pharmacokinetics and prediction of dopamine D2 receptor occupancy after multiple doses of RBP-7000, a new sustained-release formulation of risperidone, in schizophrenia patients on stable oral risperidone treatment. Clin Pharmacokinet 2014;53:533-43.

2. Leon J, Susce MT, Pan RM, et al. A study of genetic (CYP2D6 and $A B C B 1$ ) and environmental (drug inhibitors and inducers) variables that may influence plasma risperidone levels. Pharmacopsychiatry 2007;40:93-102.

3. Hiemke C, Bergemann N, Clement HW, et al. Consensus guidelines for therapeutic drug monitoring in Neuropsychopharmacology: update 2017. Pharmacopsychiatry 2018;51:9-62.

4. Li H F LM, Lin ZG, et al. The relationships between plasma concentration of risperidone and clinical effects. Shanghai Archives of Psychiatry 1999;11:189-92.

5. Nishikage H, Nakanishi T, Takamitsu Y, et al. Sequential changes in the plasma concentration of risperidone following intentional overdose. Clin Neuropharmacol 2002;25:307-9.

6. Springfield AC, Bodiford E. An overdose of risperidone. J Anal Toxicol 1996;20:202-3.

7. Ravin DS, Levenson JW. Fatal cardiac event following initiation of risperidone therapy. Ann Pharmacother 1997;31:867-70.

8. Lendoiro $\mathrm{E}$, de Castro A, Jiménez-Morigosa $\mathrm{C}$, et al. Usefulness of hair analysis and psychological tests for identification of alcohol and drugs of abuse consumption in driving license regranting. Forensic Sci Int 2018;286:239-44.

9. Matsuno H, Uematsu T, Nakashima M. The measurement of haloperidol and reduced haloperidol in hair as an index of dosage history. Br J Clin Pharmacol 1990;29:187-94.

10. Schneider S, Sibille E, Yegles M, et al. Time resolved analysis of risperidone and 9-hydroxy-risperidone in hair using LC/MS-MS. Journal of Chromatography B 2009;877:2589-92.

11. Sun XJ, Lin ZG, Ren JJ, et al. Determination of risperidone and its metabolite of 9-hydroxyrisperidone in hair by LC-MS/MS. Progress in Modern Biomedicine 2015;15:2221-4.

12. Shen $M$, Xiang $P$, Shen $B H$, et al. The evaluation and determination of antidepressant and antipsychotics of drug in hairs [J]. Journal of Forensic Medicine 2000;03:148-52.

13. Doherty $\mathrm{B}$, Rodriguez V, Leslie JC, et al. An electrospray ionisation tandem mass spectrometric investigation of selected psychoactive pharmaceuticals and its application in drug and metabolite profiling by liquid chromatography/electrospray ionisation tandem mass spectrometry. Rapid Commun Mass Spectrom 2007;21:2031-8.

14. Cabovska B, Cox SL, Vinks AA. Determination of risperidone and enantiomers of 9-hydroxyrisperidone in plasma by LC-MS/MS. Journal of Chromatography B 2007;852:497-504.

15. McClean S, O'Kane EJ, Smyth WF. Electrospray ionisation-mass spectrometric characterisation of selected anti-psychotic drugs and their detection and determination in human hair samples by liquid chromatography-tandem mass spectrometry. J Chromatogr $B$ Biomed Sci Appl 2000;740:141-57.

16. Ito T, Yamamoto K, Ohsawa F, et al. Association of CYP2D6 polymorphisms and extrapyramidal symptoms in schizophrenia patients receiving risperidone: a retrospective study. $J$ Pharm Health Care Sci 2018;4.

17. Arranz MJ, de Leon J. Pharmacogenetics and pharmacogenomics of schizophrenia: a review of last decade of research. Mol Psychiatry 2007;12:707-47.

18. Huang ML, Van Peer A, Woestenborghs R, et al. Pharmacokinetics of the novel antipsychotic agent risperidone and the prolactin response in healthy subjects. Clin Pharmacol Ther 1993;54:257-68.

19. Farrelly LA, Dicker P, Wynne K, et al. Adolescent risperidone treatment alters protein expression associated with protein trafficking and cellular metabolism in the adult rat prefrontal cortex. Proteomics 2014;14:1574-8.

20. Remmerie B, De Meulder M, Ariyawansa J, et al. Comparison of capillary and venous plasma drug concentrations after repeated 
administration of risperidone, paliperidone, quetiapine, olanzapine, or aripiprazole. Clin Pharmacol Drug Dev 2016;5:538-47.

21. Cassani M, Spiehler V. Analytical requirements, perspectives and limits of immunological methods for drugs in hair. Forensic Sci Int 1993;63:175-84.

22. Khorana N, Maphanta S, Lohitnavy O, et al. Comparative pharmacokinetics and bioequivalence of two tablet formulations of 2 $\mathrm{mg}$ risperidone in healthy Thai male volunteers. Int $\mathrm{J}$ Clin Pharmacol Ther 2011;49:409-14.

23. Seto K, Dumontet J, Ensom MH. Risperidone in schizophrenia: is there a role for therapeutic drug monitoring? Ther Drug Monit 2011;33:275-83.

24. HY L, Jiang XJ, Shang DW, et al. Influential factors of serum concentration/dosage ratio for schizophrenia patients after oral administration of risperidone. Chin J Clin Pharm 2018;34:117-9.

25. Sparshatt A, Taylor D, Patel MX, et al. Relationship between daily dose, plasma concentrations, dopamine receptor occupancy, and clinical response to quetiapine: a review. J Clin Psychiatry 2011;72:1108-23.

26. Wei CM, C M F, Guo RC. The relationship between dose of risperidone and the steady plasma concentrations of risperidone and 9-HYdroxyrisperidone in schizophrenic patients. Chin J Hosp Pharm 2005;25:607-8.

27. Zhao $Y$. The concntration of risperdone an 9-HYdroxyrisperidone in schizophrenic patients. Chin J Mod Drug App 2012;6.

28. Uematsu T, Matsuno H, Sato H, et al. Steady-state pharmacokinetics of haloperidol and reduced haloperidol in schizophrenic patients: analysis of factors determining their concentrations in hair. J Pharm Sci 1992;81:1008-11.

29. Patteet L, Morrens M, Maudens KE, et al. Therapeutic drug monitoring of common antipsychotics. Therapeutic Drug Monitoring 2012;34:629-51.
30. Linnet K, Johansen SS. Postmortem femoral blood concentrations of risperidone. J Anal Toxicol 2014;38:57-60.

31. Volpicelli SA, Centorrino F, Puopolo PR, et al. Determination of clozapine, norclozapine, and clozapine- $\mathrm{N}$-oxide in serum by liquid chromatography. Clin Chem 1993;39:1656-9.

32. Sommers DK, Snyman JR, van Wyk M, et al. Lack of effect of amitriptyline on risperidone pharmacokinetics in schizophrenic patients. Int Clin Psychopharmacol 1997;12:141-6.

33. Lee KS, Chae SW, Park JH, et al. Effects of single or repeated silymarin administration on pharmacokinetics of risperidone and its major metabolite, 9-hydroxyrisperidone in rats. Xenobiotica 2013;43:303-10.

34. Gaudl A, Kratzsch J, Bae YJ, et al. Liquid chromatography quadrupole linear ion trap mass spectrometry for quantitative steroid hormone analysis in plasma, urine, saliva and hair. $J$ Chromatogr A 2016;1464:64-71.

35. Shen M, Xiang P. Fundamentals and applications of hair analysis. Beijing: science press, 2010: 1-13.

36. Wang X, Johansen SS, Nielsen MKK, et al. Hair analysis in toxicological investigation of drug-facilitated crimes in Denmark over a 8-year period. Forensic Sci Int 2018;285:e1-12.

37. Khelfi A, Azzouz M, Abtroun R, et al. Determination of chlorpromazine, haloperidol, levomepromazine, olanzapine, risperidone, and sulpiride in human plasma by liquid chromatography/tandem mass spectrometry (LC-MS/MS). Int J Anal Chem 2018;2018:1-13.

38. Liu D, Li S Z X, et al. Clinical and application in forensic medicine research of hair analysis. China Foreign Medical Treatment 2010;29:178-9.

39. Spiehler V. Hair analysis by immunological methods from the beginning to 2000. Forensic Sci Int 2000;107:249-59.



Xiujia SUN obtained a master's degree from Qiqihar University in China in 2008. She had worked in Pharmaceutical Analysis sector of Wuxi New Drug Research and Development Ltd as a researcher from 2008 to 2011. Currently, she is working as a research assistant in the biochemical laboratory of Shanghai Mental Health Center. Her research interest includes clinical detection of serum concentration. 